\title{
Martin GEIGER et Antoine PÉCOUD, The Politics of International Migration Management
}

\section{Victor Piché}

\section{(2) OpenEdition \\ Journals}

Édition électronique

URL : https://journals.openedition.org/remi/5409

DOI : $10.4000 /$ remi.5409

ISSN : $1777-5418$

Éditeur

Université de Poitiers

Édition imprimée

Date de publication : 1 juin 2011

Pagination : 172-177

ISBN : 979-10-90426-00-9

ISSN : 0765-0752

\section{Référence électronique}

Victor Piché, "Martin GEIGER et Antoine PÉCOUD, The Politics of International Migration Management », Revue européenne des migrations internationales [En ligne], vol. 27 - $n^{\circ} 1$ | 2011, mis en ligne le 30 août 2011, consulté le 14 avril 2022. URL : http://journals.openedition.org/remi/5409 ; DOI : https://doi.org/ 10.4000/remi.5409

Ce document a été généré automatiquement le 14 avril 2022.

(C) Université de Poitiers 


\title{
Martin GEIGER et Antoine PÉCOUD, The Politics of International Migration Management
}

\author{
Victor Piché
}

\section{RÉFÉRENCE}

Martin GEIGER et Antoine PÉCOUD (ed.),The Politics of International Migration

Management, Palgrave, Macmillon, 2010, 305 p., ISBN : 9780230294882.

\section{La gestion des migrations internationales : un nouveau paradigme?}

1 Les années 2000 ont vu apparaître un nouveau paradigme en matière de politiques migratoires. Ce paradigme est largement connu maintenant sous le vocable anglosaxon "migration management", que nous traduisons par gestion migratoire (ou approche gestionnaire des migrations), même si le terme "management» fait davantage référence à une philosophie globale d'organisation et de gestion, d'abord associée aux entreprises, mais par la suite appliquée à un ensemble de domaines. Selon le fondateur de cette approche appliquée aux migrations internationales, Bimal Gosh, la gestion migratoire signifie gérer pour atteindre des objectifs plus ordonnés, prévisibles et humains, grâce à une gouvernance globale du régime migratoire. La gestion migratoire est basée sur une prémisse de base, à savoir que la migration, bien gérée, peut être positive pour tout le monde, à savoir les pays d'origine, les pays de destination et les migrants eux-mêmes (d'où le fameux slogan « win-win-win »).

2 Pour plusieurs critiques, comme en font foi les treize chapitres du livre dirigé par Martin Geiger et Antoine Pécoud, la gestion migratoire constitue un paradigme qui tente de diffuser une approche globale hégémonique présentant la migration comme 
une caractéristique normale du monde globalisé d'aujourd'hui. De plus, cette approche a émergé essentiellement dans le contexte historique de l'Union européenne préoccupée par la migration irrégulière considérée comme une menace et difficile à gérer compte tenu de l'extension des frontières prévues dans les traités et conventions.

3 En pratique et sur le terrain, la gestion migratoire met en place trois séries de mesures : 1) un contrôle plus efficace des frontières, y compris des mesures de "détérriorisation" visant à intercepter les migrants avant leur accès à l'Union européenne ; 2) la signature d'accords avec les pays tiers et les pays de transit afin de les amener à mettre un terme aux migrations irrégulières et de permettre le renvoi des migrants interceptés dans ces pays; 3) et l'élaboration de programme de codéveloppement en partenariat avec les pays de transit et/ou d'émigration.

4 Enfin, au cœur de cette approche se trouvent les organisations internationales, surtout l'Organisation Internationales des Migrations (OIM), qui jouent un rôle central dans la diffusion du discours de l'approche gestionnaire et qui se voient souvent dans la position de poursuivre les objectifs migratoires que les pays de l'Union européenne trouvent souvent trop controversés et préfèrent alors les transférer à ces organisations.

\section{Le discours}

5 Ce sont ces thèmes qu'abordent enfin de façon critique les différents chapitres du livre The Politics of International Migration Management. Contrairement aux nombreux livres collectifs, celui-ci présente un fil conducteur unifié avec une perspective multidisciplinaire : sociologie, géographie, science politique, droit et anthropologie. Il faut souligner en particulier l'apport de cette dernière à double titre : méthodologique, par des points de vue venant de l'intérieur des organisations (observation participante), et théorique, par l'introduction du concept foucaldien de "gouvernementalité ».

6 Les deux premiers chapitres présentent de façon succincte et claire en quoi consiste l'approche globale de la gestion des migrations internationales. Martin Geiger et Antoine Pécoud (chapitre 1) présentent l'argument central du livre. Selon eux, il y a trois tendances différentes dans le concept de gestion des migrations : 1) il s'agit d'une notion mobilisée par des acteurs, ici surtout les organisations intergouvernementales, dont l'Organisation Internationale des Migrations (OIM), le Haut Commissariat des Nations Unies pour les réfugiés $(\mathrm{HCR})$ et le Centre international pour le développement de politiques migratoires (ICMPD) ; 2) cette approche fait référence à des pratiques généralisées dans le domaine des politiques migratoires, comme les mesures de luttes contre le trafic des êtres humains, la formation des fonctionnaires dans le domaine du contrôle des frontières et de la lutte contre la migration irrégulière et le rôle de plus en plus central joué par l'Agence européenne pour la gestion de la coopération opérationnelle aux frontières extérieures (FRONTEX); 3) et enfin l'approche de la gestion des migrations constitue un ensemble discursif sur ce qu'est la migration et comment elle devrait être conceptualisée. Pour les deux auteurs, l'approche gestionnaire est technocratique et apolitique et évacue les dimensions de pouvoir et de conflits. Enfin, même si le discours gestionnaire fait systématiquement référence aux droits des migrants et des migrantes, les initiatives en ce sens n'ont pas débouché sur des engagements contraignants pour les États, rendant inopérantes ces références. 
7 Dans le chapitre 2, Sara Kalm poursuit le processus de déconstruction du discours gestionnaire. Ce discours, selon l'auteure, recouvre trois recommandations: 1) libéraliser la migration, mais de façon ordonnée et non dans le sens de la libre circulation, et cela à travers une amélioration des techniques de gestion et de coopération internationale (ici, l'autorité de l'État en matière de contrôle n'est pas contestée) ; 2) encourager davantage la migration circulaire, d'une part, en favorisant la circulation des cerveaux et, d'autre part, en comblant les emplois délaissés par les natifs ; 3) développer les subjectivités des migrants pour qu'ils se sentent davantage responsables en les façonnant comme agents de développement. On parle ainsi d'autorégulation, en lien explicite avec la notion de "gouvernementalité "suggérée par Michel Foucault. Plusieurs autres chapitres y font explicitement référence (voir par exemple les chapitres de Geiger et Pécoud, de Kasparek et de Bakker).

\section{Un paradigme européocentrique}

8 William Walter, s'interrogeant sur la politique migratoire de l'Union européenne, montre qu'en fait c'est l'immigration illégale qui constitue l'aspect le plus crucial du discours gestionnaire et est à la base de l'élaboration d'une politique européenne commune. En fait, la politique contre l'immigration illégale est tout orientée vers des questions de sécurité. Les nouvelles directions de la politique européenne visent à instaurer des partenariats avec les pays tiers, ce qu'il appelle la sécurisation du développement. Ce faisant, la politique européenne est basée sur la distinction entre l'immigration légale et illégale. Le discours anti-immigration illégale a comme objectif de restaurer la confiance dans la migration de travail (légale). Selon Walter, cette approche est une attaque aux droits de la personne et peut avoir l'effet contraire sur l'opinion publique en associant migration et criminalité et insécurité. La définition de la migration considère l'immigration illégale comme étant externe à l'UE (d'où les mesures de contrôles des frontières, visas, etc.), alors que plusieurs (parfois la majorité) des migrants dits illégaux entrent d'abord de façon autorisée et sont donc d'origine interne.

9 Un des mécanismes de contrôle établi concerne Frontex, l'Agence européenne pour la gestion de la coopération opérationnelle aux frontières extérieures. Bernd Kasparek, dans son chapitre, montre comment cette agence intervient de plus en plus souvent pour sécuriser les frontières, interventions qui couvrent quatre volets :1) au-delà de la frontière : ce sont les zones de départ où sont mis en place les premiers filtres (visas, etc.) ; 2) à travers la frontière (" across the border ») : ce sont les zones entourant la frontière ; 3) à la frontière, zones classiques où se trouvent les postes et les officiers ; 4) et enfin à l'intérieur de l'UE. Frontex participe aussi aux opérations d'extériorisation consistant par exemple à empêcher les bateaux de partir.

\section{Rôle critique des organisations internationales}

\section{L'Organisation Internationale des Migrations (OIM)}

10 Quatre chapitres analysent spécifiquement le rôle de l'Organisation Internationale des Migrations (OIM). En effet, comme le documente Fabian Georgi dans son chapitre, après le HCR, l'OIM est la plus grande organisation intergouvernementale dans le domaine des migrations internationales et l'acteur le plus important dans la diffusion de 
l'approche gestionnaire. Elle est en grande partie dépendante des pays riches, son budget provenant à $72 \%$ des pays du G7 (totalisant 33,4 millions US\$ en 2008). Comme on le verra dans les études de cas, l'oIM joue également un rôle important dans le renforcement des capacités des États en matière de formulation de politiques migratoires. Au slogan officiel de l'OIM « Pour le bénéfice de tous ", l'auteur renvoie sa propre formulation « Pour le bénéfice de quelques-uns». En effet, l'auteur conclut que l'OIM travaille surtout pour le bénéfice des pays industrialisés (les pays donateurs), au détriment des réfugiés et des travailleurs migrants à travers ses activités de contrôle. Des millions de travailleurs sont ir-régularisés grâce aux lois élaborées par l'OIM et plusieurs retours dits volontaires sont en fait des déportations.

11 L'information dans le discours global constitue un outil clé. Selon ce discours, elle permet aux migrants de faire des décisions sur la base des faits et à l'opinion publique de se rendre compte des aspects positifs de la migration, bref, l'information comme outil d'« empowerment » selon l'OIM. Dans son chapitre, Antoine Pécoud examine les campagnes d'information de l'OIM, campagnes qui sont surtout axées sur la lutte contre le trafic des êtres humains et la migration irrégulière. Ces campagnes s'adressent uniquement aux migrants et présentent un portrait noir de la migration, en insistant sur les risques d'exploitation. En fait, les campagnes visent à ce que les gens restent chez eux et s'inscrivent dans des stratégies de contrôle de la migration et sont organisées dans les pays d'origine. L'auteur conclut que ces campagnes d'information font la promotion d'une culture d'immobilité dans les pays d'émigration et de l'autorégulation (à la Foucault).

Deux études de cas permettent de voir concrètement l'oIM à l'œuvre dans ses stratégies de promotion de politiques migratoires visant les pays tiers. Dans son chapitre, Martin Geiger examine le cas de l'Albanie. Comme important pays producteur de migrants, l'Albanie est un bel exemple de mise sur pied de mesures visant à dénationaliser et européaniser la politique migratoire. Il s'agit en fait d'amener le pays à jouer un plus grand rôle dans la réduction de l'émigration. En 2004, l'Albanie adopte une stratégie migratoire nationale qui, selon l'auteur, est en fait le résultat de l'intervention de l'OIM, qui a joué le rôle de quasi-gouvernement dans l'élaboration de la politique.

La Mauritanie est un autre exemple de pays de transit qui a développé des stratégies migratoires largement importées de l'extérieur et sous le leadership de l'OIM. Dans leur chapitre, Philippe Poutignat et Jocelyne Sreiff-Fénart montrent comment le gouvernement mauritanien a été amené à répondre aux demandes de l'UE pour contrôler les flux migratoires, grâce à une forte intervention des organisations internationales (OIM, HCR, BIT) qui ont veillé à transférer les catégories cognitives et les cadres analytiques utilisés au Nord. La première étape dans le processus de formulation des politiques a été la construction d'un discours autour de la «crise " migratoire liée aux migrations vers les Îles Canaries. La coopération entre l'Espagne et la Mauritanie a ainsi permis la création d'un centre de transit à Nouadhibou. La politique migratoire ainsi constituée vise donc à lutter contre la migration illégale des migrants de transit, venant du Mali, Sénégal, Guinée, Cameroun, Côte d'Ivoire, Gambie et Togo. Les auteurs font une place importante aux résistances internes à ces politiques, mais concluent néanmoins que le rôle des groupes internes mauritaniens comme partenaires demeure plutôt vide. 


\section{Le Centre international pour le développement de politiques migratoires (ICMPD)}

Basé à Vienne, le Centre international pour le développement de politiques migratoires (ICMPD) a été fondé en 1993 par l'Autriche et la Suisse et regroupe onze États membres. Selon Sabine Hess, les objectifs du centre sont, comme pour l'OIM, de renforcer les capacités régulatrices des états européens et de promouvoir l'européanisation des politiques migratoires. Plus petit que le HCR et l'OIM, ce centre demeure influent dans la conceptualisation et la formulation des politiques migratoires de l'Union européenne. L'ICMPD s'est particulièrement impliqué avec les pays de l'Afrique du nord et de l'Afrique subsaharienne afin de préparer l'espace pour l'externalisation de la politique migratoire, au-delà des frontières de l'Union européenne.

\section{Refoulement préventif}

15 La pratique du refoulement préventif est de plus en plus utilisée et présentée comme une mesure respectant le principe de non refoulement prévu dans la Convention de Genève en matière de protection des réfugiés. Chira Marchetti, dans son chapitre montre comment l'Italie s'est engagée sur cette voie. Selon elle, l'interception en 2009 par les gardes côtières de 227 migrants sur trois bateaux et leur renvoi en Libye constituent un tournant historique en matière de refoulement préventif. La justification de ces interventions découle de la redéfinition des frontières maritimes comme se situant à une douzaine de miles nautiques de la terre ferme. Le refoulement préventif n'est possible qu'à travers des accords bilatéraux et multilatéraux (l'Italie a signé vingt-neuf traités à ce jour). L'auteure conclut qu'avec son nouveau modèle et ses relations avec la Libye, l'Italie est en avant-garde en matière de refoulement préventif.

L'Australie est également très avancée dans cette voie comme en fait foi son approche nommée "Solution du Pacifique ». Analysée par Claire Inder, l'approche australienne vise à « gérer » l'arrivée de demandeurs d'asile et de migrants irréguliers par la mer, de façon à être cohérente avec les obligations de l'Australie en matière de droit international des réfugiés. Pour ce faire, certaines îles au nord du pays sont exclues de la zone de migration et le traitement des arrivées irrégulières s'effectue dans des centres de réception « offshore » dans les îles de Nauru et Manus (Papua New Guinée), grâce à une série d'accords bilatéraux. L'auteur qualifie cette approche d'hyperlégaliste.

\section{L'exemple américain}

17 L'Amérique du nord est généralement peu présente dans les débats et discussions sur l'approche gestionnaire des migrations. Le chapitre de Matt Bakker est donc intéressant en ce qu'il montre que les États-Unis se situent également dans la mouvance de ce nouveau paradigme. Ainsi, il documente la transnationalisation croissante des politiques migratoires entre les États-Unis et le Mexique, incluant le même assemblage "transferts monétaires/co-développement». Les discussions bilatérales se sont particulièrement intensifiées sous la présidence de Vincente Fox (2000-2006) et se dont déroulées autour de la revendication d'un " ALENA Plus », sur le modèle de l'Union européenne. Dans cette vision, "ALENA Plus» envisage une Amérique du nord où les contrôles frontaliers disparaîtraient graduellement. Les revendications portent sur cinq points spécifiques : 1) la légalisation de la population 
mexicaine irrégulière vivant aux USA ; 2) l'élimination des restrictions sur les visas ; 3) l'accès élargi aux visas de travail temporaire ; 4) la coopération sur la sécurité ; 5) et la promotion du développement au Mexique, surtout dans les régions pourvoyeuses de migrants. Ces discussions ont été arrêtées après les attaques du 11 septembre 2001. Actuellement, l'emphase est mise sur les transferts monétaires et le co-développement, tout en tentant de rendre plus efficaces les contrôles frontaliers au nom de la sécurité et de la lutte contre les migrations irrégulières.

\section{Conclusion}

18 En guise de conclusion, nous avons choisi le chapitre de Giuila Scalettaris sur l'approche globale du HCR développée pour faire face au cas spécifique de la mobilité afghane en Iran et au Pakistan. Selon l'auteure, le cadre analytique actuel en matière de mobilité internationale est basé sur la distinction entre réfugiés et migrants. Or - et tous les auteurs du livre ont insisté sur ce point - la réalité est plus complexe et cette distinction est loin d'être nette. Ceci est particulièrement vrai pour les migrations des populations afghanes vers l'Iran et le Pakistan, où se mêlent migrations de refuges, économiques, circulaires et d'établissement. Le cadre analytique global propose quatre catégories de personnes, demandant chacune des solutions spécifiques: 1) ceux qui sont volontaires pour le retour (les migrants de retour futurs): la solution ici est le rapatriement volontaire et la ré-insertion ; 2) ceux ayant de longues durées de séjour et qui se sont intégrés de facto: la solution pour eux passe par l'accès sécuritaire à la résidence de longue durée ; 3) ceux ayant besoin de protection et qui ne peuvent retourner dans leur pays par crainte de persécution (en fait la catégorie de population généralement concernée par le HCR) : pour eux, il faut prévoir des mécanismes d'asile améliorés et des programmes de protection; 4) et les migrants et migrantes économiques pour lesquels il faut prévoir des politiques offrant des voies légales d'entrée et d'établissement légaux.

19 C'est ce cadre qui, selon nous, devrait servir de base à toutes formulations politiques, au-delà d'une approche strictement gestionnaire et hyper-légaliste, affranchies de préoccupations purement sécuritaires mettant l'emphase sur les mécanismes de contrôles. Le livre dirigé par Martin Geiger et Antoine Pécoud, pionnier en matière de critiques de l'approche gestionnaire, est un bon point de départ pour déconstruire le nouveau paradigme et proposer des solutions davantage en lien avec la vie concrète des migrants et des migrantes.

\section{AUTEURS}

\section{VICTOR PICHÉ}

Professeur honoraire de démographie, Université de Montréal. Chercheur associé, Chaire

Oppenheimer en droit international public, Université McGill 